\title{
Dysregulated long noncoding RNAs (IncRNAs) in hepatocellular carcinoma: implications for tumorigenesis, disease progression, and liver cancer stem cells
}

Xiaoqi Huo ${ }^{1}$, Shuanglin Han ${ }^{1,2}$, Guang Wu', Olivier Latchoumanin', Gang Zhou', Lionel Hebbard ${ }^{3}$, Jacob George ${ }^{1}$ and Liang Qiao ${ }^{1 *}$

\begin{abstract}
Hepatocellular carcinoma (HCC) is one of the most common malignant tumours with a poor prognosis worldwide. While early stage tumours can be treated with curative approaches such as liver transplantation or surgical resection, these are only suitable for a minority of patients. Those with advanced stage disease are only suitable for supportive approaches and most are resistant to the conventional chemotherapy or radiotherapy. Liver cancer stem cells (LCSCs) are a small subset of cancer cells with unlimited differentiation ability and tumour forming potential. In order to develop novel therapeutic approaches for HCC, we need to understand how the cancer develops and why treatment resistance occurs. Using high-throughput sequencing techniques, a large number of dysregulated long noncoding RNAs (IncRNAs) have been identified, and some of which are closely linked to key aspects of liver cancer pathology, progression, outcomes and for the maintenance of cancer stem cell-like properties. In addition, some IncRNAs are potential biomarkers for HCC diagnosis and may serve as the therapeutic targets. This review summarizes data recently reported IncRNAs that might be critical for the maintenance of the biological properties of LCSCS.
\end{abstract}

Keywords: Hepatocellular carcinoma (HCC), Liver cancer stem cells (LCSCS), Long non-coding RNAs (InCRNAs)

\section{Background}

Although more than $70 \%$ of the whole human genome is transcriptionally very active, only less than $2 \%$ of the transcripts are eventually translated into proteins $[1,2]$. Long noncoding RNAs (lncRNAs) are a subclass of functional ncRNAs (tRNA and rRNA are not included in this review); they are over 200 nucleotides in size and are incapable of encoding protein [3, 4]. lncRNAs may share some characteristics of mRNAs [4]. For instance, lncRNAs are transcribed by RNA polymerase II; they are $5^{\prime}$ capped, equipped with $3^{\prime}$ polyA (polyadenylate) tail and consist of multiple exons $[4,5]$. Initially, IncRNAs were thought to be junk or transcriptional noise since they are not well conserved across species and because expression levels were relatively lower compared with mRNAs [5-8].

\footnotetext{
* Correspondence: liang.qiao@sydney.edu.au

${ }^{1}$ Storr Liver Centre, Westmead Institute for Medical Research, University of

Sydney and Westmead Hospital, Westmead, NSW 2145, Australia

Full list of author information is available at the end of the article
}

However, recent studies suggest that lncRNAs play a key role in many biological processes such as $\mathrm{X}$ chromosome inactivation, cell cycle regulation, and cardiac development $[4,5,9,10]$. IncRNAs are also involved in the development of many diseases. For example, using microarray analysis, a list of dysregulated (either up-regulated or down-regulated) IncRNAs have been identified in many tumour types such as prostate, liver, lung, and breast cancer [11-18]. Broadly, lncRNAs can be classified as either oncogenic or tumor-suppressive [19].

Hepatocellular carcinoma (HCC) is a leading cause of cancer related death worldwide [20,21], with more than 500,000 new cases reported every year [22]. Early stage HCC can be effectively treated by liver transplantation or curative surgery, but for advanced cases, the therapeutic strategies are limited [23, 24]. Tumour recurrence and disease relapse after therapy, as well as drug resistance are the critical issues leading to poor prognosis $[24,25]$. 
Within the liver tumour bulk, a small group of cells known as liver cancer stem cells (LCSCs) are considered to be responsible for the initiation, recurrence and drug resistance of $\mathrm{HCC}[26,27]$. How LCSCs are regulated at the molecular level is not well understood. Knowledge of the key regulators of LCSC behaviour would facilitate the development of more effective therapeutic approaches IIn this regard, increasing evidence has shown that lncRNAs may be involved in the regulation of the biological function of LCSCs. For example, multiple lncRNAs are expressed aberrantly in LCSCs compared to non-cancer stem cells [28, 29], while some lncRNAs are required for the self-renewal and tumour propagation of LCSCs [3032 ], or are closely associated with the clinico-pathological features [8, 33, 34]. However, the precise function of lncRNAs in LCSCs is poorly defined. This review summarizes current understanding of the lncRNAs and their implications for HCC and LCSCs.

\section{Origin and classification of IncRNA}

Unlike mRNA, lncRNAs are not very well conserved across species [8, 35]. These RNAs may derive from several sources: (1) insertion of a transposable element; (2) duplication of noncoding RNA; (3) transformation from a previous protein coding gene; and (4) chromatin rearrangement [36-38] (Fig. 1). In fact, lncRNAs can be generated either from protein coding genes or non-protein coding genes. A common way of categorizing lncRNAs is based on the relative position with respect to the closest protein coding gene in the genome. In this way, lncRNAs can be classified into the following five categories: (1) intergenic IncRNAs: transcribed from the space between two protein coding genes; (2) intronic lncRNAs: incorporated inside the intron of a protein coding gene; (3) sense lncRNAs: transcribed from the sense strand of a protein coding gene; (4) antisense lncRNAs: transcribed from the antisense strand of a protein coding gene, usually overlapping the introns or exons of the sense strand; and (5) bidirectional lncRNAs: transcribed in an opposite direction with respect to the nearby protein coding gene and located within $1 \mathrm{~kb}$ from the promoter of the protein coding gene [8, 38] (Fig. 2).

\section{IncRNAs are involved in HCC through multiple mechanisms}

lncRNAs are present in either nucleus or cytoplasm, and they can interact with DNA, RNA or protein [39, 40]. Through this, they modulate the expression and stability of their downstream targets at epigenetic, transcriptional and post-transcriptional levels [41] (Fig. 3).

At the epigenetic level, lncRNAs can regulate the expression of target gene via recruiting chromatin modifying factors to alter chromatin structure [42]. For example, an up-regulated lncRNA CDKN2B antisense RNA 1 (ANRIL) in $\mathrm{HCC}$ has been demonstrated to promote cell proliferation; its expression level is positively associated with the Barcelona Clinic Liver Cancer (BCLC) stage [43]. This ncRNA is mostly present in the nucleus where it silences the function of Kruppel-like factor 2 (KLF2) through recruiting polycomb repressive complex 2 (PRC2) to the promoter region of KLF2 resulting in H3K27 trimethylation and KLF2 silencing [43].

IncRNAs are able to modulate the expression of nonprotein coding genes such as microRNAs. In this aspect, down-regulation of a lncRNA H19 has been shown to play an important role in HCC metastasis [44]. H19 directly binds hnRNP U/PCAF/RNA polII complex resulting in the active transcription of the miR-200 family by acetylating histone $\mathrm{H} 3$ of its promoter [44].

IncRNAs have also been found to function at a posttranscriptional level to control the progression of HCC via regulating alternative splicing and mRNA stability [41]. For example, an up-regulated lncRNA termed metastasis-associated lung adenocarcinoma transcript 1 (MALAT1) in HCC increases the expression of splicing factor SRSF1 (serine and arginine rich splicing factor 1) through activating Wnt pathway, causing dys-regulated
(1)

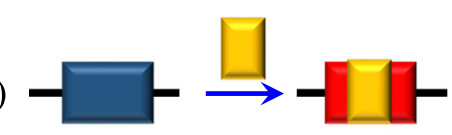

(3)

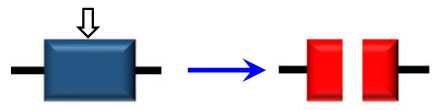

(4)

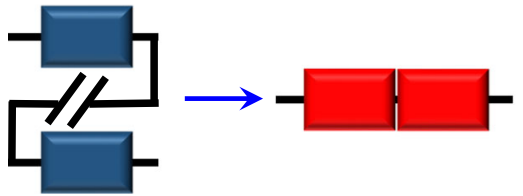

(2)

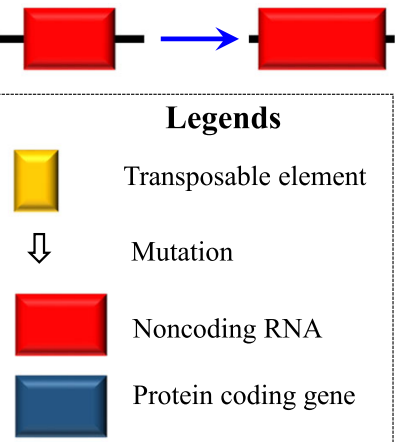

Fig. 1 Multiple origins of IncRNAs. IncRNA may derive from (1) insertion of a transposable element; (2) duplication in non-coding RNA; (3) a previous protein coding gene; (4) chromatin rearrangement [36-38] 


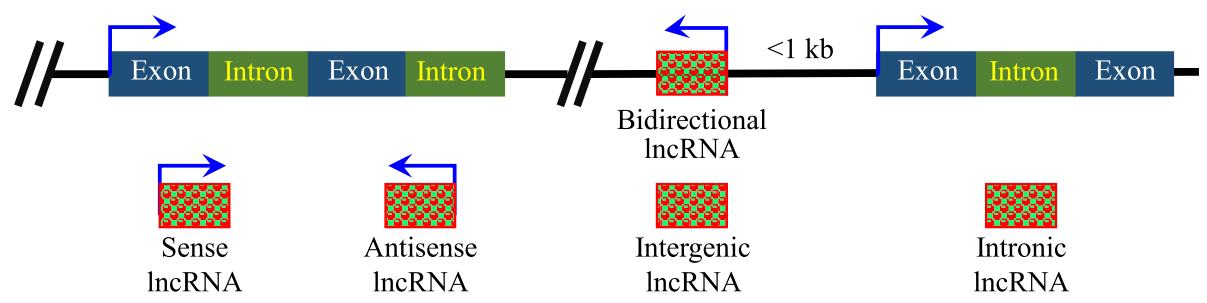

Fig. 2 Classification of IncRNAs. Multiple type of IncRNAs have been identified, including (1) Sense IncRNA: transcribed from the sense strand of a protein coding gene; (2) Antisense IncRNA: transcribed from the antisense strand of a protein coding gene, usually overlapping the introns or exons of the sense strand; (3) Intergenic IncRNA: transcribed from the space between two protein coding genes; (4) Intronic IncRNA: incorporated inside of the introns of protein coding genes; (5) Bidirectional IncRNA: transcribed in an opposite direction with respect to the nearby protein coding gene and located within $1 \mathrm{~kb}$ of the promoter of the protein coding gene $[8,38]$

apoptosis and alternative splicing of S6 kinase1 (S6 K1), which further activates the mTOR pathway, and ultimately resulting in HCC progression [45].

Antisense lncRNAs can affect the stability of mRNAs. For example, an up-regulated antisense lncRNA termed PCNA antisense RNA 1 (PCNA-AS1) in HCC has been shown to prompt tumor growth by forming a RNA duplex with proliferating cell nuclear antigen (PCNA), leading to alteration of PCNA structure and increase in its stability [46].

Studies over the recent years have also revealed that lncRNAs can act as competing endogenous RNAs (ceRNAs) and play important roles in HCC [47]. In this perspective, an over-expressed lncRNA in HCC termed colon cancer associated transcript 1 (CCAT1) was found to competitively bind to let-7 (as evidenced by increased expression of let-7 target genes including high mobility group AT-hook2 and c-Myc), leading to increased proliferation and migration of HCC cells [48]. Similarly, a newly identified lncRNA unigene56159 also functions as a ceRNA [49]. Unigene56159 is significantly up-regulated in HBV-related HCC tissues, and is required for epithelialmesenchymal transition (EMT) and migration of HCC cells by acting as a ceRNA for miR-140-5p to increase the expression of Slug [49].

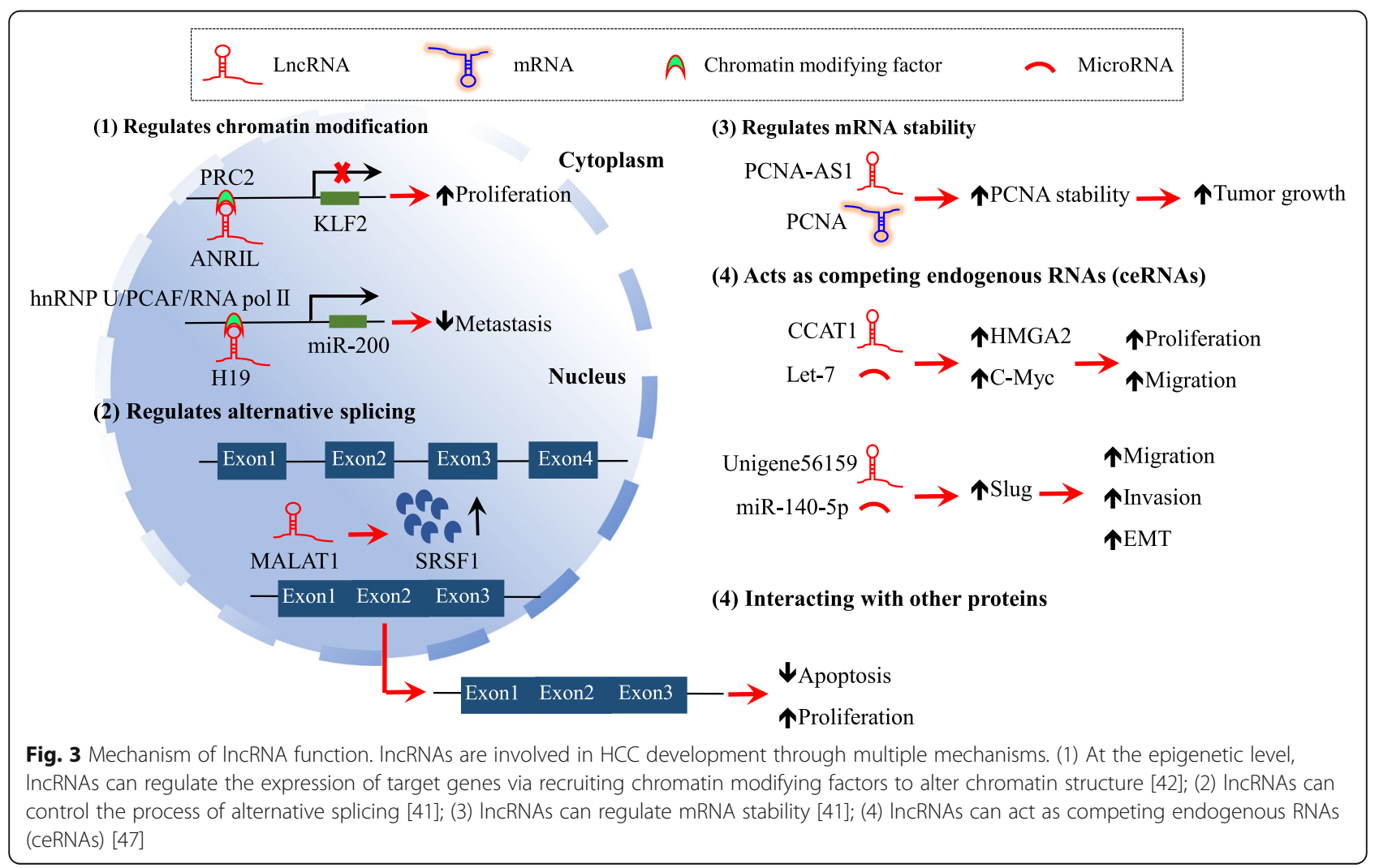




\section{HCC associated IncRNA}

By microarray or next-generation sequencing, a large number of significantly dysregulated lncRNAs have been identified in HCC tumour tissues as compared with adjacent non-tumorous liver [50]. Below, we will summarize some of the deregulated lncRNAs that may play key roles in HCC development and progression, and describe their correlation with the clinical outcomes of HCC. The reported data may provide new sights for the early diagnosis and for therapeutic targeting.

\section{Dysregulated IncRNAs implicated in the regulation of HCC proliferation}

Deregulated control of the ability to proliferate and undergo apoptosis is an important feature of cancers [51]. Many lncRNAs are involved in the regulation of uncontrolled cell growth in HCC. TP73 antisense RNA 1 (TP73-AS1) is a lncRNA that is overexpressed in HCC tissues compared to the adjacent non-tumorous liver [52]. TP73-AS1 competes with high mobility group box 1 (HMGB1) to bind miR200a and activates HMGB1/ RAGE axis thereby promoting the proliferation of $\mathrm{HCC}$ cells [52]. As such, knockdown of TP73-AS1 by siRNA leads to impaired proliferation and colony formation of HepG2 and HCCLM3 cells [52].

Colon cancer associated transcript 2 (CCAT2) is an oncogenic lncRNA transcribed from chromosome 8q24.21 [53]. Up-regulation of CCAT2 has been identified in several HCC cell lines (HepG2, Hep3B, HCCLM3 and Huh7) and tumour tissues [53]. Overexpression of CCAT2 in HepG2 and Huh7 cells significantly promotes their proliferation while apoptosis is reduced [53]. Opposite results were observed when CCAT2 was knocked down in these cells [53].

lncRNAs may perform their biological functions via interacting with other proteins. For example, overexpression of a lncRNA termed ultraconserved element 338 (uc.338) was shown to promote the proliferation of HCC cells in a BMI1-dependent manner as confirmed by RNA pull down and RNA immunoprecipitation assays [54].

In addition to up-regulated lncRNAs, some significantly down-regulated lncRNAs play critical roles in HCC. For example, significantly reduced expression of lncRNA BRAF-activated noncoding RNA (BANCR) has been observed in HCC tissues and HCC cell lines (Huh7, HepG2 and SMMC-7721). Consequently, forced overexpression of IncRNA BANCR in these cells inhibits their proliferation in vitro and in vivo [55]. Another down-regulated lncRNA termed long noncoding RNA FTX (lnc-FTX) in $\mathrm{HCC}$ can modulate the proliferation of $\mathrm{HCC}$ and is closely related with the prognosis [56]. Thus, lnc-FTX itself functions as a tumor suppressor by interacting with minichromosome maintenance protein 2 (MCM2), suppressing the proliferation of HCC cells. In addition, lnc-FTX may function as a ceRNA by binding to miR-374a, thereby inhibiting the activity of Wnt pathway and decrease in EMT and invasion [56]. Patients with low hepatic lnc-FTX have reduced survival and early recurrence suggesting that lnc-FTX might serve as a biomarker of prognosis [56].

\section{LncRNAs involved in the regulation of epithelial to mesenchymal transition in HCC}

Previous studies have confirmed that epithelial to mesenchymal transition (EMT) contributes to multiple stages of cancer development such as progression, invasion and metastasis [57]. Increasing evidence now demonstrates that many lncRNAs play a role in triggering EMT during the pathogenesis of HCC. For instance, up-regulation of a IncRNA urithelial carcinoma-associated 1 (UCA1) in HCC tissues has been closely linked to tumour size, invasion and tumour progression [58]. In vitro studies also suggested significant up-regulation of lncRNA UCA1 in multiple HCC cell lines (Huh7, SK-Hep-1, MHCC97H and MHCC97L) [58]. By using gain- and lose-of-function analysis in $\mathrm{MHCC} 97 \mathrm{H}$ and $\mathrm{MHCC} 97 \mathrm{~L}$ cells, a positive correlation between the expression of lncRNA UCA1 and EMT markers such as Snail2 and Vimentin was identified, as well as a negative correlation between the expression of lncRNA UCA1 and E-cadherin [58]. These data suggest that overexpression of lncRNA UCA1 may promote HCC development by driving EMT [58]. As mentioned above, CCAT2 is a lncRNA up-regulated in HCC and high level of lncRNA CCAT2 correlates with poorer patient survival and a worse prognosis [59]. This lncRNA not only promotes the proliferation of HCC cells, but also activates EMT via up-regulating Snail2 [59]. Similarly, two other lncRNAs Sprouty4-Intron 1 (SPRY4-IT1) and small nucleolar RNA host gene 20 (SNHG20) that are overexpressed in HCC have been shown to prompt EMT via upregulating the transcription factors twist 1 and Vimentin while reducing the expression of E-cadherin [60-62].

\section{Role of IncRNAs in HCC drug resistance}

Resistance to chemotherapy or radiotherapy is a serious hurdle in the clinical management of patients with HCC. Indeed, resistance to previously first-line anticancer agents like adriamycin is responsible for a large portion of treatment failure in patients with advanced liver cancer [63-65]. Multiple studies have revealed that some IncRNAs may be mechanistically responsible for the development of anticancer drug resistance in HCC.

Taurine up-regulated gene 1 (TUG1) is an overexpressed lncRNA present in adriamycin-resistant HCC tissues and cell lines (SMMC-7721/ADM and HepG2/ADM). In turn, siTUG1 mediated knockdown of TUG1 restored the sensitivity of resistant cells to adriamycin [65]. Mechanistically, TUG1 was found to regulate the expression of Pglycoprotein (P-gp) and multidrug-resistance 1 (MDR1) 
which are closely related to the development of drug resistance [65].

Although the mechanism of drug resistance is not well understood, protective autophagy has been proposed to be involved in chemoresistance [66, 67]. Highly upregulated in liver cancer (HULC) RNA is another commonly up-regulated lncRNA in HCC [67]. Up-regulation of HULC has been linked to the development of resistance of HCC cells to oxaliplatin, 5-fluorouracil (5-FU) and pirarubicin (THP) via activating autophagy by protecting the degradation of silent information regulator 1 (SIRT1) [67]. This finding suggests that HULC may be a novel target for improving the chemosensitivity [67].

\section{Role of IncRNAs in the regulation of liver cancer stem cells (LCSCs)}

Although LCSCs only represent a small portion of cells within HCCs, they are a critical subset of cells that are believed to account for the initiation, progression, recurrence, metastasis and treatment resistance of liver cancer $[26,27]$. An increasing number of dysregulated lncRNAs have been identified in LCSCs. Among them, a range of lncRNAs directly regulate the stem cell features or indirectly regulate LCSCs via interacting with other key signaling pathways, such as Wnt and IL6/STAT3 pathways [68]. Some identified lncRNAs involved in the regulation of the function of LCSCs are summarized below.

\section{HOX antisense intergenic RNA (HOTAIR)}

HOX antisense intergenic RNA (HOTAIR) is a $2.2 \mathrm{~kb}$ lncRNA localized to chromosome $12 \mathrm{q} 13.13$ within Homeobox C (HOXC) cluster [69, 70]. The oncogenic role of HOTAIR has been demonstrated in multiple cancers such as breast cancer, melanoma, and colorectal cancer [19, 71, 72]. Up-regulation of HOTAIR has also been identified in HCC [73] and LCSCs where it has been linked to the expansion of stem cells. Mechanistically, HOTAIR reduces the recruitment of P300, RNA polymerase II (RNApol II) and cAMP responsive element binding protein 1 (CREB) to the promoter of SET Domain-Containing Protein 2 (SETD2) thereby suppressing SETD2 activity [73]. Reduced SETD2 activity impairs the binding to histone 3 (H3) and subsequently leads to reduced trimethylation on histone $\mathrm{H} 3$ thirtysixth lysine (H3K36me3), resulting in reduced formation of the H3K36me-hMSH2-hMSH6-SKP2 complex. These changes eventually disrupt DNA damage repair mechanisms and cause microsatellite instability (MSI) [73].

\section{Lnc-DILC}

Lnc-DILC is a recently identified novel lncRNA with tumor suppressive activity [28]. By microarray and qRT-PCR analysis, reduced expression of lnc-DILC has been observed in LCSCs [28]. Using RNA in situ hybridization, lnc-DILC was found to reside in both the nucleus and cytoplasm [28]. In vivo and in vitro experiments demonstrate that lncDILC suppresses the expansion of LCSCs via inhibiting interleukin 6 (IL-6)/JAK2/STAT3 signaling [28]. Using multivariate analysis, it was found that lnc-DILC may serve as an independent prognostic marker for HCC. Thus, patients with low hepatic expression have shorter survival time and a higher frequency of tumour recurrence than those with higher lnc-DILC levels [28]. These data indicate that lnc-DILC could serve as a potential target for HCC therapy and as a biomarker for monitoring outcome.

\section{LnCBRM}

LINCR-0003 (lncBRM) is a lncRNA overexpressed in HCC tissues and LCSCs; it is required for the maintenance of the stemness features and for the biological properties of LCSCs through Yes-associated protein 1 (YAP1) signaling [29]. LncBRM is present in the cell nucleus and is localized between actin beta like 2 (ACTBL2) and polo like kinase 2 (PLK2) on chromosome 5, with a size of 1321 nt containing six exons [29]. By RNA pulldown assay, IncBRM interactt with BRM and this interaction facilitates the formation of BRG1embedded BAF (BRG1-associated factor) complex. The latter can then bind to the promoter region of YAP1 and along with the enrichment of KLF4, ultimately leads to activation of YAP1 signaling, a critical pathway for the maintenance of stemness features of LCSCs [29].

\section{Cancer up-regulated drug resistant (CUDR)}

Cancer up-regulated drug resistant (CUDR) is an upregulated oncogenic lncRNA in LCSCs [74]. CUDR is present in both the nucleus and cytoplasm and exerts its biological function by promoting the growth of LCSCs through up-regulating the expression of CyclinD1 and down-regulating phosphatase and tensin homolog (PTEN). This promotes the formation of a CUDR-CyclinD1 complex [74] that inhibits the methylation of H19 promoter, increases H19 transcription and increases the formation of telomerase reverse transcriptase-telomerase RNA component (TERT-TERC) complex, thereby leading to increased telomerase activity [74]. In addition, the CUDR-CyclinD1 complex can be recruited by CCCTC-binding factor (CTCF) to form a CUDR-CyclinD1-CTCF complex which can engage the promoter of c-Myc leading to increased cMyc expression [74]. These changes synergistically contribute to the expansion of LCSCs [74].

\section{LncTCF7}

Wnt signaling has been confirmed to play an important role in the self-renewal and propagation of CSCs [75]. Recently, an intergenic lncRNA termed lncTCF7 was found to activate the Wnt pathway in LCSCs by modulating its nearby gene expression [30]. IncTCF7 is present in the 
nucleus and is up-regulated in HCCs and LCSCs [30]. lncTCF7 sustains the self-renewal of LCSCs by recruiting SWItch/Sucrose Non-Fermentable (SWI/SNF) complex to the promoter of its nearby protein coding gene transcription factor 7 (TCF7) leading to increased TCF7 transcription and activation of Wnt signaling pathway [30]. Of note, lncTCF7 can barely be detectable in normal liver, making it a promising biomarker for the diagnosis and therapeutic targeting of liver cancer [30].

\section{LncRNA for $\beta$-catenin methylation (Inc- $\beta$-Catm)}

lncRNA for $\beta$-catenin methylation (lnc- $\beta$-Catm) is an overexpressed lncRNA identified in LCSCs [31]. Inc- $\beta$-Catm is localized to the chromosome 1q between interferon regulatory factor 2 binding protein 2 (IRF2BP2) and translocase of outer mitochondrial membrane 20 (TOMM20) genes with a size of $2281 \mathrm{nt}$ and contains two exons [31]. lnc- $\beta$ Catm regulates the self-renewal of LCSCs via activating the Wnt signaling [31]. Inc- $\beta$-Catm mediates the formation of a RNA-protein complex consisting of $\beta$-catenin and enhancer of zeste homolog 2 (EZH2) in which $\beta$-catenin is methylated by EZH2 [31]. This leads to increased stability of $\beta$ catenin and eventually triggers activation of Wnt signaling pathway [31]. Patients with high levels of lnc- $\beta$-Catm have a relatively poorer prognosis [31].

\section{LncRNA calmodulin binding transcription activator 1 (CAMTA1)}

lncRNA CAMTA1 is highly expressed in HCCs and LCSCs [32]. Using gain- and loss-of-function studies, it was found that IncRNA CAMTA1 was essential for the maintenance of the CSC properties in HCC through regulating the expression of calmodulin binding transcription activator 1 (CAMTA1) [32]. Direct binding of lncCAMTA1 to the CAMTA1 promoter changes the chromatin structure and inhibits the transcription of CAMTA1 as confirmed by chromatin isolation by the RNA purification Assay (CHIRP) [32]. Inhibition of CAMTA1 facilitates the maintenance of cancer stem cell-like properties [32]. The essential oncogenic role of IncRNA CAMTA1 in liver cancer was further supported by the finding that patients with higher levels of IncRNA CAMTA1 have shorter survival and an increased rate of early recurrence [32].

\section{LnCRNA DANCR}

IncRNA differentiation antagonizing non-protein coding RNA (DANCR) is a lncRNA with significant up-regulation in HCC and LCSCs $[76,77]$. DNACR is involved in the maintenance and regulation of the stemness feature of LCSCs [77]. More mechanistic studies have shown that DNACR promotes HCC via acting as a ceRNA since it competitively impairs the binding between CTNNB1 ( $\beta$-catenin) and miRNA (miR-214, miR-320a and miR-199a), leading to increased CTNNB1 stability [77]. Patients with
DANCR overexpression often show increased rates of tumour recurrence and poor survival [77].

\section{Plasmacytoma variant translocation 1 (PVT1)}

lncRNA plasmacytoma variant translocation 1 (PVT1) is an oncofetal lncRNA with increased expression and an oncogenic role in many solid tumours (e.g., cervical, gastric, colorectal, esophageal, pancreatic, non-small cell lung, ovarian, bladder and thyroid cancers as well as in acute promyelocytic leukemia) [78-87]. Overexpression of lncPVT1 has been found not only in pre-cancerous liver conditions such as inactivated hepatic stellate cells (HSCs) and fibrotic liver tissues [88], but also in adult HCC [89]. In the development of liver fibrosis, lncPVT1 competitively binds to miR-152 to promote liver fibrosis via the Hedgehog $(\mathrm{Hh})$ and EMT pathway [90]. In the development of liver cancer, both in vivo and in vitro studies demonstrate that lncPVT1 can prompt the proliferation and stem cell-like features of liver cancer cells by stabilizing an RNA binding protein named nucleolar protein (NOP2) [89]. Clinically, lncPVT1 is positively associated with tumour size, tumour stage and HBV infection [89].

\section{Clinical implications of IncRNAs in the early diagnosis and survival prediction}

Early diagnosis and intervention remains the most effective approach to achieving a better outcome for HCC [91]. In this aspect, sensitive and specific markers for early diagnosis are urgently required. Alpha fetal protein (AFP) has been widely used as a diagnostic marker for HCC, but its sensitivity and specificity are suboptimal [92, 93]. IncRNAs with a significantly aberrant expression profile in HCC tissues as opposed to healthy liver may hold great potential as diagnostic markers [94]. Some lncRNAs may be stably present in body fluids such as plasma and their expression levels are consistently associated with the progression of HCC [94]. These lncRNAs hold potential value as diagnostic markers for HCC [94].

HULC is an up-regulated IncRNA in HCC. Meanwhile, high levels of HULC has been detected in the plasma of HCC patients with higher Edmondson grades. Hence, it was proposed that HULC may possess diagnostic value [95]. Through screening blood samples of HCC patients by microarray, an expression profile of circulating lncRNAs has been established [96]. By qRT-PCR assay, three novel lncRNAs (RP11-160H22.5, XLOC-014172 and LOC149086) were shown to be significantly up-regulated in liver and matched plasma from HCC patients [96]. Furthermore, increased expression of XLOC-014172 and LOC149086 was found to be predictors for HCC metastasis [96].

The above lncRNAs are stably present in patient serum and possess moderate specificity (73\%) and sensitivity (82\%) for HCC [96]. Hence, they have potential either alone or more likely in combination with other variables as biomarkers for diagnosis and monitoring. 


\section{Prospective and conclusion}

With the application of high-throughput sequencing, a tremendous number of newly identified dysregulated lncRNAs are reported every year. At present, more than 30,000 dysregulated lncRNAs have been identified in humans and the number is still increasing as a result of improved screening technology $[8,97]$. Of the large number of lncRNAs identified so far, some (e.g., TUG1 and HOTAIR) have been shown to play very important roles in multiple aspects of liver cancer [65, 73] such as tumourigenesis, tumour recurrence and drug resistance (Table. 1). Some lncRNAs (e.g., IncBRM, IncTCF7 and PVT1) play regulatory roles in the maintenance of stemness properties of LCSCs and hence may have a role in tumour initiation [29, 30, 89] (Table. 1). Other lncRNAs (e.g., HULC) have potential to serve as diagnostic markers [95]. However, detailed clarification of the roles of lncRNAs in liver cancer is lacking, partially because lncRNAs have more complicated functions and multiple modes of functions [98]. Besides, unlike mRNAs, IncRNAs are not

Table 1 Deregulated IncRNAs in HCC

\begin{tabular}{|c|c|c|c|c|}
\hline & IncRNAs & Function & Mechanisms & References \\
\hline \multirow[t]{21}{*}{ Up-regulated } & ANRIL & Promotes cell proliferation & Interacts with PRC2 to silent KLF2 & {$[43]$} \\
\hline & MALAT1 & $\begin{array}{l}\text { Induces apoptosis, activates } \\
\text { mTOR pathway }\end{array}$ & Increases the splicing factor SRSF1 & {$[45]$} \\
\hline & PCNA-AS1 & Promotes tumour growth & Forms a duplex with PCNA, increases PCNA stability & {$[46]$} \\
\hline & CCAT1 & $\begin{array}{l}\text { Promotes cell proliferation } \\
\text { and migration }\end{array}$ & Binds let-7 as a ceRNA & {$[48]$} \\
\hline & Unigene56159 & Induces EMT and migration & Binds miR-140-5p as a ceRNA & {$[49]$} \\
\hline & TP73-AS1 & Promotes cell proliferation & $\begin{array}{l}\text { Competes with HMGB1 to bind miR200a, } \\
\text { activate HMGB1/RAGE axis }\end{array}$ & {$[52]$} \\
\hline & CCAT2 & $\begin{array}{l}\text { Promote cell proliferation, } \\
\text { EMT and reduces apoptosis }\end{array}$ & Up-regulates Snail2 & {$[53,59]$} \\
\hline & uc.338 & Promotes cell proliferation & Interacts with BMI1 & {$[54]$} \\
\hline & UCA1 & Promotes invasion and EMT & Interacts with miR-203, regulates the expression of Snail2 & {$[58]$} \\
\hline & SPRY4-IT1 & Promotes EMT & $\begin{array}{l}\text { Up-regulates twist1 and Vimentin, reduces the } \\
\text { expression of E-cadherin }\end{array}$ & {$[60]$} \\
\hline & SNHG20 & Promotes EMT & $\begin{array}{l}\text { Up-regulates twist1 and Vimentin, reduces the } \\
\text { expression of E-cadherin }\end{array}$ & {$[61,62]$} \\
\hline & TUG1 & Induces drug resistance & Regulates the expression of P-gp and MDR1 & {$[65]$} \\
\hline & HULC & $\begin{array}{l}\text { Induces resistance to oxaliplatin, } \\
\text { 5-FU and THP }\end{array}$ & Activates autophagy & {$[67]$} \\
\hline & HOTAIR & Damages DNA repair and induces MSI & $\begin{array}{l}\text { Reduces the recruitment of P300, RNA Pol II and } \\
\text { CREB to SETD2 promoter }\end{array}$ & {$[73]$} \\
\hline & IncBRM & $\begin{array}{l}\text { Maintains stemness and properities } \\
\text { of LCSCs }\end{array}$ & Interacts with BRM and activates YAP1 signalling & {$[29]$} \\
\hline & CUDR & Promotes growth of LCSCs & Up-regulates CyclinD1, down-regulated PTEN & {$[74]$} \\
\hline & IncTCF7 & $\begin{array}{l}\text { Promotes self-renewal and } \\
\text { propagation of CSCs }\end{array}$ & Recruits SWI/SNF complex, activates Wnt pathway & {$[30]$} \\
\hline & Inc- $\beta$-catenin & Regulates self-renewal of LCSCs & $\begin{array}{l}\text { Mediates the formation of } \beta \text {-catenin -EZH2 } \\
\text { complex, activates Wnt signalling }\end{array}$ & {$[31]$} \\
\hline & IncRNA CAMTA1 & Maintains CSC properties & Regulates the expression of CAMTA1 & {$[32]$} \\
\hline & InCRNA DANCR & Maintains stemness of LCSCs & $\begin{array}{l}\text { Impairs interaction between CTNNB1 and } \\
\text { miRNA as a ceRNA }\end{array}$ & {$[77]$} \\
\hline & PVT1 & $\begin{array}{l}\text { Promotes cell proliferation and } \\
\text { stem cell-like features }\end{array}$ & Stabilizes NOP2 & {$[89]$} \\
\hline \multirow[t]{4}{*}{ Down-regulated } & $\mathrm{H} 19$ & Mediates metastasis of $\mathrm{HCC}$ & $\begin{array}{l}\text { Binds hnRNP U/PCAF/RNA Pol II complex to } \\
\text { increase the expression of miR-200 family }\end{array}$ & {$[44]$} \\
\hline & BANCR & Promotes cell proliferation & & {$[55]$} \\
\hline & Inc-FTX & Modulate cell proliferation & $\begin{array}{l}\text { Interacts with MCM2 and binds miR-374a } \\
\text { as a ceRNA }\end{array}$ & {$[56]$} \\
\hline & Inc-DILC & Promotes expansion of LCSCs & Inhibits IL-6/JAK2/STAT3 & [28] \\
\hline
\end{tabular}


conserved across the species making it difficult for translating the data from the animal models to humans [35].

In terms of clinical applications, although some lncRNAs (e.g.,HULC) have shown their potential in the early diagnosis and prognosis prediction for patients with liver cancer [95], large scale clinical studies in liver cancer patients with various etiologies are needed to further validate their efficacy, and more studies are needed to explore the lncRNAs that might serve as the more specific and sensitive markers for the diagnosis and therapy for liver cancer.

\begin{abstract}
Abbreviations
5-FU: 5-fluorouracil; ACTBL2: actin beta like 2; AFP: alpha fetal protein; ANRIL: CDKN2B antisense RNA 1; BAF: BRG1-associated factor; BANCR: BRAF-activated noncoding RNA; BCLC: Barcelona Clinic Liver Cancer; CAMTA1: calmodulin binding transcription activator 1; CCAT1: colon cancer associated transcript 1; CCAT2: colon cancer associated transcript 2; ceRNA: competing endogenous RNAs; CHIRP: chromatin isolation by RNA purification Assay; CREB: CAMP responsive element binding protein 1; CTCF: CCCTC-binding factor; CUDR: cancer up-regulated drug resistant; DANCR: differentiation antagonizing non-protein coding RNA; EMT: epithelial-mesenchymal transition; EZH2: enhancer of zeste homolog 2; H3: histone 3; H3K36me3: histone H3 thirty-sixth lysine; HCC: Hepatocellular carcinoma; Hh: Hedgehog; HMGB1: high mobility group box 1; HOTAIR: HOX antisense intergenic RNA; HOXC: Homeobox C; HSCs: hepatic stellate cells; HULC: highly upregulated in liver cancer; IL-6: interleukin 6; IRF2BP2: interferon regulatory factor 2 binding protein 2; KLF2: Kruppel-like factor 2; LCSCs: Liver cancer stem cells; IncRNAs: long noncoding RNAs; Inc- $\beta$-Catm: IncRNA for $\beta$-catenin methylation; MALAT1: metastasis-associated lung adenocarcinoma transcript 1; MCM2: minichromosome maintenance protein 2; MDR1: multidrug-resistance 1; MSI: microsatellite instability; NOP2: nucleolar protein; PCNA: proliferating cell nuclear antigen; PCNA-AS1: PCNA antisense RNA 1; P-gp: P-glycoprotein; PLK2: polo like kinase 2; PRC2: polycomb repressive complex 2; PTEN: phosphatase and tensin homolog; PVT1: plasmacytoma variant translocation 1; RNApol II: RNA polymerase II; S6 K1: S6 kinase 1; SETD2: SET Domain-Containing Protein 2; SIRT1: silent information regulator 1; SNHG20: small nucleolar RNA host gene 20; SPRY4-IT1: Sprouty4-Intron 1; SRSF1: serine and arginine rich splicing factor 1; SWI/SNF: SWItch/Sucrose Non-Fermentable; TCF7: transcription factor 7; TERT-TERC: telomerase reverse transcriptase-telomerase RNA component; THP: pirarubicin; TOMM20: translocase of outer mitochondrial membrane 20; TP73-AS1: TP73 antisense RNA 1; TUG1: Taurine up-regulated gene 1; UCA1: urithelial carcinoma-associated 1:YAP1: Yes-associated protein 1
\end{abstract}

\section{Acknowledgements}

This work was partially supported by the Robert W. Storr Bequest to the Sydney Medical Foundation, University of Sydney.

\section{Availability of data and materials}

Not applicable.

\section{Authors' contributions}

All authors read and approved the final manuscript.

\section{Ethics approval and consent to participate}

Not applicable.

\section{Consent for publication}

All authors agreed on the manuscript.

\section{Competing interests}

The authors declare that they have no competing interests.

\section{Publisher's Note}

Springer Nature remains neutral with regard to jurisdictional claims in published maps and institutional affiliations.

\section{Author details}

'Storr Liver Centre, Westmead Institute for Medical Research, University of Sydney and Westmead Hospital, Westmead, NSW 2145, Australia.

2Department of Gastroenterology, The Second Affiliated Hospital of Dalian Medical University, Dalian, Liaoning Province 116027, China. ${ }^{3}$ Department of Molecular and Cell Biology, Centre for Comparative Genomics, The Centre for Biodiscovery and Molecular Development of Therapeutics, James Cook University, Australian Institute of Tropical Health and Medicine, QLD, Townsville 4811, Australia.

Received: 25 August 2017 Accepted: 16 October 2017 Published online: 23 October 2017

\section{References}

1. Birney E, Stamatoyannopoulos JA, Dutta A, Guigó R, Gingeras TR, Margulies $\mathrm{EH}$, et al. Identification and analysis of functional elements in 1\% of the human genome by the ENCODE pilot project. Nature. 2007;447:799-816.

2. Djebali S, Davis CA, Merkel A, Dobin A, Lassmann T, Mortazavi A, et al. Landscape of transcription in human cells. Nature. 2012;489:101-8.

3. Laurent GS, Wahlestedt C, Kapranov P. The landscape of long noncoding RNA classification. Trends Genet. 2015;31:239-51.

4. Devaux Y, Zangrando J, Schroen B, Creemers EE, Pedrazzini T, Chang C-P, et al. Long noncoding RNAs in cardiac development and ageing. Nat Rev Cardiol. 2015;12:415-25.

5. Ma L, Bajic VB, Zhang Z. On the classification of long non-coding RNAs. RNA Biol. 2013;10:924-33.

6. Wang J, Zhang J, Zheng H, Li J, Liu D, Li H, et al. Mouse transcriptome: neutral evolution of 'non-coding'complementary DNAs. Nature. 2004;431:1-2.

7. Kung JT, Colognori D, Lee JT. Long noncoding RNAs: past, present, and future. Genetics. 2013;193:651-69.

8. Derrien T, Johnson R, Bussotti G, Tanzer A, Djebali S, Tilgner $\mathrm{H}$, et al. The GENCODE V7 catalog of human long noncoding RNAs: analysis of their gene structure, evolution, and expression. Genome Res. 2012;22:1775-89.

9. Penny GD, Kay GF, Sheardown SA, Rastan S, Brockdorff N. Requirement for Xist in X chromosome inactivation. Nature. 1996;379:131.

10. Mourtada-Maarabouni M, Hedge VL, Kirkham L, Farzaneh F, Williams GT. Growth arrest in human T-cells is controlled by the non-coding RNA growth-arrest-specific transcript 5 (GAS5). J Cell Sci. 2008;121:939-46.

11. Huarte M, Rinn JL. Large non-coding RNAs: missing links in cancer? Hum Mol Genet. 2010;19:R152-R61.

12. Pickard MR, Williams GT. Regulation of apoptosis by long non-coding RNA GAS5 in breast cancer cells: implications for chemotherapy. Breast Cancer Res Treat. 2014;145:359-70.

13. Pickard M, Mourtada-Maarabouni M, Williams G. Long non-coding RNA GAS5 regulates apoptosis in prostate cancer cell lines. BBA-MOL Basis Dis. 1832;2013:1613-23.

14. Zhao X, Liu M, Zhang J, Zhang R, Zhang Q, Tan X. Long noncoding RNA CPS1-IT1 suppresses cell proliferation and metastasis in human lung cancer. Oncol Res. 2017;25:373-80.

15. Yu L, Fang F, Lu S, Li X, Yang Y, Wang Z. Lncrna-hit promotes cell proliferation of non-small cell lung cancer by association with E2f1. Cancer Gene Ther. 2017;24:221-6.

16. Zhang M, Wang W, Li T, Yu X, Zhu Y, Ding F, et al. Long noncoding RNA SNHG1 predicts a poor prognosis and promotes hepatocellular carcinoma tumorigenesis. Biomed Pharmacother. 2016;80:73-9. https://doi.org/10.1016/ j.biopha.2016.02.036

17. LC X, Chen QN, Liu XQ, Wang XM, Chang QM, Pan Q, et al. Up-regulation of LINC00161 correlates with tumor migration and invasion and poor prognosis of patients with hepatocellular carcinoma. Oncotarget. 2017; 10 . 18632/oncotarget.17040.

18. Huarte M. The emerging role of IncRNAs in cancer. Nat Med. 2015;21:1253-61.

19. Qi P, Du X. The long non-coding RNAs, a new cancer diagnostic and therapeutic gold mine. Mod Pathol. 2013;26:155-65.

20. Torre LA, Bray F, Siegel RL, Ferlay J, Lortet-Tieulent J, Jemal A. Global cancer statistics, 2012. CA Cancer J Clin. 2015;65:87-108.

21. Ferlay J, Soerjomataram I, Dikshit R, Eser S, Mathers C, Rebelo M, et al. Cancer incidence and mortality worldwide: sources, methods and major patterns in GLOBOCAN 2012. Int J Cancer. 2015;136:E359-E86.

22. Mittal S, El-Serag HB. Epidemiology of HCC: consider the population. J Clin Gastroenterol. 2013;47:S2. 
23. Llovet JM. Liver cancer: time to evolve trial design after everolimus failure. Nat Rev Clin Oncol. 2014;11:506-7.

24. Forner A, Llovet JM, Bruix J. Hepatocellular carcinoma. Lancet (London, England). 2012;379:1245-55. https://doi.org/10.1016/s0140-6736(11)61347-0.

25. Tanaka S, Arii S. Molecular targeted therapy for hepatocellular carcinoma in the current and potential next strategies. J Gastroenterol. 2011;46:289-96.

26. Kreso A, Dick JE. Evolution of the cancer stem cell model. Cell Stem Cell. 2014;14:275-91.

27. Song K, Wu J, Jiang C. Dysregulation of signaling pathways and putative biomarkers in liver cancer stem cells (review). Oncol Rep. 2013;29:3-12. https://doi.org/10.3892/or.2012.2082.

28. Wang X, Sun W, Shen W, Xia M, Chen C, Xiang D, et al. Long non-coding RNA DILC regulates liver cancer stem cells via IL-6/STAT3 axis. J Hepatol. 2016;64:1283-94.

29. Zhu P, Wang Y, Wu J, Huang G, Liu B, Ye B, et al. LncBRM initiates YAP1 signalling activation to drive self-renewal of liver cancer stem cells. Nat Commun. 2016;7:13608. https://doi.org/10.1038/ncomms13608.

30. Wang Y, He L, Du Y, Zhu P, Huang G, Luo J, et al. The long noncoding RNA IncTCF7 promotes self-renewal of human liver cancer stem cells through activation of Wnt signaling. Cell Stem Cell. 2015;16:413-25.

31. Zhu P, Wang Y, Huang G, Ye B, Liu B, Wu J, et al. Lnc-[beta]-Catm elicits EZH2-dependent [beta]-catenin stabilization and sustains liver CSC selfrenewal. Nat Struct Mol Biol. 2016;23:631-9.

32. Ding L-J, Li Y, Wang S-D, Wang X-S, Fang F, Wang W-Y, et al. Long noncoding RNA IncCAMTA1 promotes proliferation and cancer stem cell-like properties of liver cancer by inhibiting CAMTA1. Int J Mol Sci. 2016;17:1617.

33. Mang Y, Li L, Ran J, Zhang S, Liu J, Li L, et al. Long noncoding RNA NEAT1 promotes cell proliferation and invasion by regulating hnRNP A2 expression in hepatocellular carcinoma cells. OncoTargets Ther. 2017;10:1003-16. https://doi.org/10.2147/OTT.S116319.

34. Ni W, Zhang Y, Zhan Z, Ye F, Liang Y, Huang J, et al. A novel IncRNA uc.134 represses hepatocellular carcinoma progression by inhibiting CUL4Amediated ubiquitination of LATS1. J Hematol Oncol. 2017;10(91) https://doi. org/10.1186/s13045-017-0449-4

35. Pang KC, Frith MC, Mattick JS. Rapid evolution of noncoding RNAs: lack of conservation does not mean lack of function. Trends Genet. 2006:22:1-5.

36. Felekkis K, Voskarides K. Genomic elements in health, disease and evolution: junk DNA: springer New York; 2015.

37. Kapusta A, Kronenberg Z, Lynch VJ, Zhuo X, Ramsay L, Bourque G, et al. Transposable elements are major contributors to the origin, diversification, and regulation of vertebrate long noncoding RNAs. PLoS Genet. 2013;9:e1003470.

38. Ponting CP, Oliver PL, Reik W. Evolution and functions of long noncoding RNAs. Cell. 2009;136:629-41.

39. Moran VA, Perera RJ, Khalil AM. Emerging functional and mechanistic paradigms of mammalian long non-coding RNAs. Nucleic Acids Res. 2012; 40:6391-400.

40. Yoon JH, Abdelmohsen $\mathrm{K}$, Gorospe M. Functional interactions among microRNAs and long noncoding RNAs. Semin Cell Dev Biol. 2014;34:9-14. https://doi.org/10.1016/j.semcdb.2014.05.015.

41. Shi X, Sun M, Liu H, Yao Y, Song Y. Long non-coding RNAs: a new frontier in the study of human diseases. Cancer Lett. 2013:339:159-66.

42. Campos El, Reinberg D. Histones: annotating chromatin. Annu Rev Genet. 2009;43:559-99.

43. W-mC M-d H, F-z Q, Xia R, Sun M, T-p X, Yin L, et al. Long non-coding RNA ANRIL is upregulated in hepatocellular carcinoma and regulates cell apoptosis by epigenetic silencing of KLF2. J Hematol Oncol. 2015;8:1-14.

44. Zhang L, Yang F, Yuan J-h, Yuan S-x, Zhou W-p, Huo X-s, et al. Epigenetic activation of the MiR-200 family contributes to H19-mediated metastasis suppression in hepatocellular carcinoma. Carcinogenesis. 2013;34:577-86.

45. Malakar P, Shilo A, Mogilevsky A, Stein I, Pikarsky E, Nevo Y, et al. Long noncoding RNA MALAT1 promotes hepatocellular carcinoma development by SRSF1 upregulation and mTOR activation. Cancer Res. 2017;77:1155-67.

46. Yuan S-X, Tao Q-F, Wang J, Yang F, Liu L, Wang L-L, et al. Antisense long noncoding RNA PCNA-AS1 promotes tumor growth by regulating proliferating cell nuclear antigen in hepatocellular carcinoma. Cancer Lett. 2014;349:87-94.

47. Salmena $L$, Poliseno $L$, Tay $Y$, Kats $L$, Pandolfi Pier P. A ceRNA hypothesis: the Rosetta stone of a hidden RNA language? Cell. 2011;146:353-8. https:// doi.org/10.1016/j.cell.2011.07.014.

48. Deng L, Yang SB, FF X, Zhang JH. Long noncoding RNA CCAT1 promotes hepatocellular carcinoma progression by functioning as let-7 sponge. J Exp Clin Canc Res. 2015;34:18. https://doi.org/10.1186/s13046-015-0136-7.
49. Lv J, H-x F, X-p Z, Lv P, J-y F, Zhang Y, et al. Long non-coding RNA Unigene56159 promotes epithelial-mesenchymal transition by acting as a ceRNA of miR-140-5p in hepatocellular carcinoma cells. Cancer Lett. 2016;382:166-75.

50. Zhu J, Liu S, Ye F, Shen Y, Tie Y, Zhu J, et al. The long noncoding RNA expression profile of hepatocellular carcinoma identified by microarray analysis. PLoS One. 2014;9:e101707.

51. Hanahan D, Weinberg RA. Hallmarks of cancer: the next generation. Cell. 2011;144:646-74.

52. Li S, Huang Y, Huang Y, Fu Y, Tang D, Kang R, et al. The long non-coding RNA TP73-AS1 modulates HCC cell proliferation through miR-200adependent HMGB1/RAGE regulation. J Exp Clin Canc Res. 2017;36:51.

53. Zhou N, Si Z, Li T, Chen G, Zhang Z, Qi H. Long non-coding RNA CCAT2 functions as an oncogene in hepatocellular carcinoma, regulating cellular proliferation, migration and apoptosis. Oncol Lett. 2016;12:132-8.

54. Bo C, Li N, Li X, Liang X, An Y. Long noncoding RNA uc. 338 promotes cell proliferation through association with BMI1 in hepatocellular carcinoma. Hum Cell. 2016:29:141-7.

55. Yu X, Zhang G, Zhao P, Cui M, Wang C, He Y. Downregulation of a long noncoding RNA BANCR contributes to proliferation and metastasis of hepatocellular carcinoma cancer cells in vitro and in vivo. Int J Clin Exp Patho. 2016:9:3304-12.

56. Liu F, Yuan J, Huang J, Yang F, Wang T, Ma J, et al. Long noncoding RNA FTX inhibits hepatocellular carcinoma proliferation and metastasis by binding MCM2 and miR-374a. Oncogene. 2016;35:5422-34.

57. Berx G, Raspé E, Christofori G, Thiery JP, Sleeman JP. Pre-EMTing metastasis? Recapitulation of morphogenetic processes in cancer. Clin Exp Metastasis. 2007;24:587-97.

58. Xiao J-N, Yan T-H, Yu R-M, Gao Y, Zeng W-L, Lu S-W, et al. Long non-coding RNA UCA1 regulates the expression of Snail2 by miR-203 to promote hepatocellular carcinoma progression. J Cancer Res Clin. 2017:143:981-90.

59. Xu Y, Wang B, Zhang F, Wang A, Du X. Hu P, et al. long non-coding rna ccaT2 is associated with poor prognosis in hepatocellular carcinoma and promotes tumor metastasis by regulating snail2-mediated epithelialmesenchymal transition. OncoTargets Ther. 1191;2017:10.

60. Zhou M, Zhang X-Y, Overexpression YX. Of the long non-coding RNA SPRY4-IT1 promotes tumor cell proliferation and invasion by activating EZH2 in hepatocellular carcinoma. Biomed Pharmacother. 2017:85:348-54.

61. Zhang D, Cao C, Liu L, Up-regulation WD. Of LncRNA SNHG20 predicts poor prognosis in hepatocellular carcinoma. J Cancer. 2016;7:608.

62. Liu J, Lu C, Xiao M, Jiang F, Qu L, Ni R. Long non-coding RNA SNHG20 predicts a poor prognosis for HCC and promotes cell invasion by regulating the epithelial-to-mesenchymal transition. Biomed Pharmacother. 2017:89:857-63.

63. Morii M, Fukumoto Y, Kubota S, Yamaguchi N, Nakayama Y, Yamaguchi N. Imatinib inhibits inactivation of the ATM/ATR signaling pathway and recovery from adriamycin/doxorubicin-induced DNA damage checkpoint arrest. Cell Biol Int. 2015;39:923-32.

64. Villanueva A, Llovet JM. Second-line therapies in hepatocellular carcinoma: emergence of resistance to sorafenib. Clin Cancer Res. 2012;18:1824-6.

65. Yang L, Du Y, Yu P, Fan J, Wang X, Long WY. Non-coding RNA TUG1 regulates the development of multidrug resistance in hepatocellular carcinoma via P-gp and MDR1. Int J Clin Exp Med. 2016;9:21388-96.

66. Song J, Qu Z, Guo X, Zhao Q, Zhao X, Gao L, et al. Hypoxia-induced autophagy contributes to the chemoresistance of hepatocellular carcinoma cells. Autophagy. 2009;5:1131-44.

67. Xiong $\mathrm{H}, \mathrm{Ni} Z$, He J, Jiang S, Li X, Gong W, et al. LnCRNA HULC triggers autophagy via stabilizing SIRT1 and attenuates the chemosensitivity of HCC cells. Oncogene. 2017;36:3528-40.

68. Ji J, Wang XW. Clinical implications of cancer stem cell biology in hepatocellular carcinoma. Semin Oncol. 2012;39:461-72. https://doi.org/10. 1053/j.seminoncol.2012.05.011

69. Rinn JL, Kertesz M, Wang JK, Squazzo SL, Xu X, Brugmann SA, et al. Functional demarcation of active and silent chromatin domains in human HOX loci by noncoding RNAs. Cell. 2007;129:1311-23.

70. Gupta RA, Shah N, Wang KC, Kim J, Horlings HM, Wong DJ, et al. Long noncoding RNA HOTAIR reprograms chromatin state to promote cancer metastasis. Nature. 2010:464:1071-6.

71. Hajjari M, Salavaty AHOTAIR. An oncogenic long non-coding RNA in different cancers. Cancer Biology \& Medicine. 2015;12:1.

72. Tang L, Zhang W, Su B, Long YB. Noncoding RNA HOTAIR is associated with motility, invasion, and metastatic potential of metastatic melanoma. Biomed Res Int. 2013;2013 
73. Li H, An J, Wu M, Zheng Q, Gui X, Li T, et al. LncRNA HOTAIR promotes human liver cancer stem cell malignant growth through downregulation of SETD2. Oncotarget. 2015;6:27847.

74. Pu H, Zheng Q, Li H, Wu M, An J, Gui X, et al. CUDR promotes liver cancer stem cell growth through upregulating TERT and C-Myc. Oncotarget. 2015; 6:40775.

75. Takebe N, Harris PJ, Warren RQ, Ivy SP. Targeting cancer stem cells by inhibiting Wnt, notch, and hedgehog pathways. Nat Rev Clin Oncol. 2011:8:97-106.

76. Ma X, Wang X, Yang C, Wang Z, Han B, Wu L, et al. DANCR Acts as a Diagnostic Biomarker and Promotes Tumor Growth and Metastasis in Hepatocellular Carcinoma. Anticancer Res. 2016;36:6389-98. 10.21873/ anticanres.11236.

77. Yuan SX, Wang J, Yang F, Tao QF, Zhang J, Wang LL, et al. Long noncoding RNA DANCR increases stemness features of hepatocellular carcinoma by derepression of CTNNB1. Hepatology. 2016;63:499-511. https://doi.org/10. 1002/hep.27893.

78. Zhang S, Zhang G, Liu J. Long noncoding RNA PVT1 promotes cervical cancer progression through epigenetically silencing miR-200b. APMIS. 2016; 124:649-58.

79. Xu M-d, Wang Y, Weng W, Wei P, Qi P, Zhang Q, et al. A positive feedback loop of IncRNA-PVT1 and FOXM1 facilitates gastric cancer growth and invasion. Clin Cancer Res. 2017;23:2071-80.

80. Li Q, Dai Y, Wang F, Hou S. Differentially expressed long non-coding RNAs and the prognostic potential in colorectal cancer. Neoplasma. 2016;63:977-83.

81. Zheng $\mathrm{X}, \mathrm{Hu}$ H, Li S. High expression of IncRNA PVT1 promotes invasion by inducing epithelial-to-mesenchymal transition in esophageal cancer. Oncology Lett. 2016;12:2357-62.

82. Xie Z, Chen X, Li J, Guo Y, Li H, Pan X, et al. Salivary HOTAIR and PVT1 as novel biomarkers for early pancreatic cancer. Oncotarget. 2016;7:25408.

83. Wan L, Sun M, Liu G-J, Wei C-C, Zhang E-B, Kong R, et al. Long noncoding RNA PVT1 promotes non-small cell lung cancer cell proliferation through epigenetically regulating LATS2 expression. Mol Cancer Ther. 2016;15:1082-94.

84. Liu E, Liu Z, Zhou Y, Mi R, Wang D. Overexpression of long non-coding RNA PVT1 in ovarian cancer cells promotes cisplatin resistance by regulating apoptotic pathways. Int J Clin Exp Med. 2015;8:20565.

85. Zeng C, Yu X, Lai J, Yang L, Chen S, Li Y. Overexpression of the long noncoding RNA PVT1 is correlated with leukemic cell proliferation in acute promyelocytic leukemia. J Hematol Oncol. 2015;8:126.

86. Zhuang C, Li J, Liu Y, Chen M, Yuan J, Fu X, et al. Tetracycline-inducible shRNA targeting long non-coding RNA PVT1 inhibits cell growth and induces apoptosis in bladder cancer cells. Oncotarget. 2015;6:41194.

87. Zhou Q, Chen J, Feng J, Wang J. Long noncoding RNA PVT1 modulates thyroid cancer cell proliferation by recruiting EZH2 and regulating thyroidstimulating hormone receptor (TSHR). Tumor Biol. 2016;37:3105-13.

88. Bai G, Yan G, Wang G, Wan P, Zhang R. Anti-hepatic fibrosis effects of a novel turtle shell decoction by inhibiting hepatic stellate cell proliferation and blocking TGF- $\beta 1 /$ Smad signaling pathway in rats. Oncol Rep. 2016;36: 2902-10.

89. Wang F, Yuan JH, Wang SB, Yang F, Yuan SX, Ye C, et al. Oncofetal long noncoding RNA PVT1 promotes proliferation and stem cell-like property of hepatocellular carcinoma cells by stabilizing NOP2. Hepatology. 2014;60: 1278-90.

90. Zheng J, Yu F, Dong P, Wu L, Zhang Y, Hu Y, et al. Long non-coding RNA PVT1 activates hepatic stellate cells through competitively binding microRNA-152. Oncotarget. 2016;7:62886.

91. Chauhan R, Lahiri N. Tissue-and serum-associated biomarkers of hepatocellular carcinoma. Biomarkers in Cancer. 2016;8:37.

92. Gervain J. Symptoms of hepatocellular carcinoma. Laboratory tests used for its diagnosis and screening. Orv Hetil. 2010;151:1415-7.

93. Di Carlo I, Mannino M, Toro A, Ardiri A, Galia A, Cappello G, et al. Persistent increase in alpha-fetoprotein level in a patient without underlying liver disease who underwent curative resection of hepatocellular carcinoma. A case report and review of the literature. World J Surg Oncol. 2012;10:79.

94. Zheng $\mathrm{C}$, Hao H, Chen L, Shao J. Long noncoding RNAs as novel serum biomarkers for the diagnosis of hepatocellular carcinoma: a systematic review and meta-analysis. Clin Transl Oncol. 2017;19:961-8.

95. Xie H, Ma H, Zhou D, Plasma HULC. As a promising novel biomarker for the detection of hepatocellular carcinoma. Biomed Res Int. 2013;2013:136106-10.

96. Tang J, Jiang R, Deng L, Zhang X, Wang K, Sun B. Circulation long noncoding RNAs act as biomarkers for predicting tumorigenesis and metastasis in hepatocellular carcinoma. Oncotarget. 2015;6:4505.
97. Hon C-C, Ramilowski JA, Harshbarger J, Bertin N, Rackham OJ, Gough J, et al. An atlas of human long non-coding RNAs with accurate $5^{\prime}$ ends. Nature. 2017;

98. Novikova IV, Hennelly SP, Sanbonmatsu KY. Sizing up long non-coding RNAs: do IncRNAs have secondary and tertiary structure? BioArchitecture. 2012;2:189-99.

\section{Submit your next manuscript to BioMed Central and we will help you at every step:}

- We accept pre-submission inquiries

- Our selector tool helps you to find the most relevant journal

- We provide round the clock customer support

- Convenient online submission

- Thorough peer review

- Inclusion in PubMed and all major indexing services

- Maximum visibility for your research

Submit your manuscript at www.biomedcentral.com/submit

) Biomed Central 